\title{
Influences of Retrieval Processes on the Spacing Effect in Free Recall
}

\author{
Arthur M. Glenberg \\ University of Wisconsin-Madison
}

\begin{abstract}
A two-process theory of the spacing effect in free recall is presented and tested. The first process, differential organization, produces a positive correlation between the spacing of the presentations of repeated words and the number of different retrieval routes that can provide access to the words. The retrieval process interacts with the differential organization to control performance. If the cues used to retrieve the words provide approximately equal access to all retrieval routes, then the function relating spacing to recall will increase monotonically. If only selected retrieval routes are used, then the spacing function will be nonmonotonic. Evidence supporting this theory is that (a) the monotonic spacing function is most robust when subjects study the list using an organizational strategy, (b) cuing and directing retrieval with input words can result in a nonmonotonic effect of spacing when subjects have used an organizational strategy, and (c) directing retrieval by instructions about the order of recall can result in a nonmonotonic effect of spacing.
\end{abstract}

The free-recall paradigm has been used extensively to investigate the effects of the spacing (lag) between the presentations of repeated items (D'Agostino \& DeRemer, 1973 ; Madigan, 1969 ; Melton, 1970 ; Underwood, 1969). The predominate finding is that items given massed presentations, when the two presentations of a repeated item are contiguous, are recalled less often than items whose presentations are distributed. In addition, many investigators have reported that

The first experiment is taken from a dissertation submitted to the University of Michigan in partial fulfillment for the requirements of the doctoral degree. Gratitude is expressed to the members of my committee: Arthur W. Melton (Chairman), Robert A. Bjork, Louis Jensen, and Robert G. Pachella. The experiment was completed while I was supported by a U.S. Public Health Service traineeship. Experiment 1 was funded by the Air Force Office of Scientific Research, under Contract No. F44602C-0038. Experiments 2, 3, and 4 were supported by U.S. Public Health Service Grant No. 1-R01MH26643-01 to A. M. Glenberg. Many thanks are due to Carol Green, Robert Gruenberg, and Rosie Zimering, who assisted in the data collection and analyses of these experiments.

Requests for reprints should be sent to Arthur M. Glenberg, Department of Psychology, University of Wisconsin, Madison, Wisconsin 53706. the amount of distribution is positively correlated with recall. This last finding, the lag effect, has been demonstrated with a variety of materials, using a large range of presentation rates and using interpresentation lags of up to 80 items. In free recall the lag effect has always increased monotonically (except for one case, Foos \& Smith, 1974, which is discussed later); increasing the lag produced increases in recall, never decreases. This fact is made particularly interesting considering the shape of the lag effect in the paired-associate literature.

Glenberg (1976) demonstrated that the shape of the lag function in the continuous paired-associate paradigm depends on the conditions of retrieval. Changing the conditions of retrieval resulted in lag functions that were monotonically increasing or nonmonotonic, first increasing then decreasing. These results were explained within an encoding variability theory along with assumptions detailing the interaction of the stored input and the retrieval cues. The purpose of this article is to extend that theory to free recall and to demonstrate that the shape of the lag function in free recall is dependent on the conditions of retrieval. 
When faced with the free-recall task, a subject associates (Anderson, 1972), organizes (Tulving, 1962), or chunks (Buschke, 1976) groups of items. The individual items in a chunk can be related on semantic, acoustic, or orthographic dimensions, or the subject can chunk items based on input contiguity (Buschke, 1976; Wallace, 1969). The chunks themselves can be arranged hierarchically. The important point is that subjects impose a structure on the free-recall list by relating groups of items. For this article I am assuming that the internal structure imposed on a list consists of overlapping groups of (perhaps interassociated) words.

The second presentation of a repeated item can be grouped with essentially the same items as at the first presentation or with different items. The major assumption of a differential organization theory of the lag effect is that the probability that a repeated item is grouped with different items on its presentations is directly related to the lag between the presentations.

When asked to recall the list, subjects access the groups of items using various retrieval cues. Once the group is accessed, the individual items can be retrieved and recalled. If all of the groups are more or less equally accessible, then the monotonically increasing lag effect is predicted. An item repeated after a long lag is associated with more different groups than an item repeated after a short lag. These multiple retrieval routes give the item repeated after a long lag an advantage in retrieval, and hence in recall.

The lag effect may not be monotonic, however, if the groups of items are not equally accessible. Specifically, suppose that the subject is provided with a recall cue composed of a word presented once, adjacent to one of the presentations, and between the two presentations of the repeated word. Suppose, further, that this cue is represented in, or can provide access to, at least some groups of items. A word repeated after a short lag (e.g., Lag 2, two items between the first and second presentations) would be associated with few groups. If the Lag 2 word happens to be associated with a group accessible by the cue word, then the Lag 2 word would be recalled. A word repeated after a moderate lag (e.g., Lag 5) would be associated with more different items than the Lag 2 word (differential organization). The probability that the Lag 5 word is associated with a group accessible by the cue is greater than the corresponding probability for the Lag 2 word. In short, the Lag 5 word should be recalled more often than the Lag 2 word.

Now consider a word repeated after a longer lag, for example, Lag 17. This word would be associated with the most different groups. These associates, however, belong to two sets of relatively nonoverlapping groups. One set is composed of the items associated with the Lag 17 word at its first presentation; the other set is composed of the items associated at the second presentation. The sets of groups are relatively nonoverlapping, since the two presentations were widely separated. The cue, which was adjacent to only one presentation, should enhance the accessibility of only one set of groups. Therefore, the number of cue-accessible groups associated with the Lag 17 word is comparable to the number of cue-accessible groups associated with the Lag 2 word. The Lag 17 word should be recalled at about the same level as the Lag 2 word, and both should be recalled less often than the Lag 5 word.

The prediction of nonmonotonicity has been discussed in relation to cuing, with input words presented adjacent to one presentation of a repeated word. The nonmonotonicity does not, however, depend on the use of input words as cues. Any method of directing retrieval to enhance the accessibility of selected portions of the internal representation of the list should produce the nonmonotonic lag effect. In Experiments 1 and 2 , retrieval is directed by cue words as described above. Cuing should result in the nonmonotonic lag effect, while free recall should result in the monotonic lag effect. In Experiment 4 , retrieval is directed by instructions concerning the order of recall.

\section{Experiment 1}

\section{Method}

Subjects. A total of 48 male and female subjects served in the experiment. All but 2 of the subjects 
were students in introductory psychology classes at the University of Michigan. These 2 subjects were drawn from the Human Performance Center subject pool and were paid $\$ 2$ for participating in the experiment.

Materials and design. Two presentation sequences were constructed. Each sequence was composed of 75 positions. The first 5 and the last 6 positions were filled with once-occurring primacy and recency words. Within the body of the list were 40 once-occurring filler words and 12 once-occurring cue words. The other 12 positions were filled with two exemplars of words repeated at each lag of 2, 5, and 17 intervening words. Each presentation of the repeated words was separated from the other repeated words by at least 5 other words. The difference between the two presentation sequences was in the order in which the repeated items were arranged in the list. The first half of the first sequence was ordered as the following: first and second presentation of the Lag 2 item, first presentation of the Lag 17 item, first and second presentations of the Lag 5 item, and then the second presentation of the Lag 17 item. The ordering of the second half of the first sequence was as follows: first presentation of the Lag 17 item, first and second presentation of the Lag 2 item, second presentation of the Lag 17 item, and then the first and second presentations of the Lag 5 item. In the second sequence, the Lag 2 and Lag 5 items switched positions. This arrangement tended to equalize the average serial position of the presentations of the repeated items.

There were 276 common, single-syllable fourletter nouns used for stimuli. These words were divided into four sets of 69 words each. The four sets of words were used to construct four lists, two of each presentation sequence. There were 6 words selected from each set to be used as the repeated words ; 12 words were selected to be used as the cue words. The remaining words were used as the primacy, filler, and recency words. Two cue words were assigned to each repeated word, one to be presented immediately after the first presentation and one immediately before the second presentation. Obvious semantic, acoustic, and orthographic similarities were avoided when assigning the cue words to their respective repeated words. The assignment of cue words to repeated words was constant throughout the experiment.

\section{Table 1}

Mean Percentage of the Repeated Words Recalled in Experiment 1

\begin{tabular}{lccc}
\hline & \multicolumn{3}{c}{ Lag } \\
\cline { 2 - 4 } Recall & 2 & 5 & 17 \\
\hline Cued & 35.4 & 41.6 & 34.4 \\
Uncued & 33.9 & 38.5 & 46.9 \\
\hline
\end{tabular}

Each subject saw all four lists. Two were recalled with cues (one of each presentation sequence), and two were uncued. The 48 subjects were divided into three groups of 16 subjects each. Each group of 16 received a different assignment of the cue-wordsrepeated-word triples to lag conditions, so that across the 48 subjects, each triple was used equally often in each lag condition. Across the 16 subjects in a group, each of the four lists was cued half of the time. In addition, the 16 subjects in a group were divided into four subgroups of 4 subjects each. These subgroups were distinguished by the cue words presented at the time of recall. For example, on the cued-recall tests, the subjects in one subgroup were cued (a) with the words following the first presentations of the first lag exemplars and (b) with the words following the first presentations of the second lag exemplars. Another subgroup was cued (a) by the words preceding the second presentations of the first lag exemplars and (b) by the words following the first presentations of the second lag exemplars.

On the cued-recall tests, the subjects were shown eight cue words. The first two words were always the last and the first words of the input list, respectively. The other six words were composed of one critical-cue word adjacent to each lag exemplar. These six words were randomly arranged for each subject.

The stimuli were projected on a screen by a Carousel slide projector. The presentation rate was one word every $2 \mathrm{sec}$. This time included the time needed to change slides (approximately $.8 \mathrm{sec}$ ). The subjects were allowed $3 \mathrm{~min}$ for written recall following the presentation of each list.

Procedure. The subjects were run in groups of 2 to 16. Each subject was handed a booklet containing five recall sheets separated by five cover sheets. The subjects were instructed to wait to the end of each list, indicated by a row of asterisks, before turning the cover sheet and beginning their recall. For the cued-recall tests, the eight cue words were printed down the left-hand column of the recall sheet. The subjects had been instructed to look at these words and to try to use the words to help them recall the other words on the list. On the uncued tests, the recall sheets were blank. For these lists the subjects had been instructed to try to recall as many of the words as they could. Following the instructions, the subjects viewed a 16-word practice list. The recall of the practice list was cued for all of the subjects. After any questions were answered, the main experiment began.

\section{Results and Discussion}

The data from the repeated items are presented in Table 1 . The data in Table 1 were analyzed in a $3 \times 4 \times 2 \times 2 \times 3$ (Group $\times$ Pattern of Cue Words Presented on CuedRecall Trial $\times$ Cued/Uncued Recall $\times$ List 
$\times$ Lag) analysis of variance. The last three factors are within-subjects variables. The rejection region was set so that alpha equals .05 for all analyses reported in this article.

There were no significant main effects. The Lag $\times$ Group interaction was significant, $F(4,72)=3.24, M S_{\mathrm{e}}=.41$. This interaction indicates that the magnitude of the spacing effect, collapsed across cuing conditions, varied across groups. The pattern of cues presented on the cued-recall tests interacted with whether recall was cued, $F(3,36)=$ $6.99, M S_{\mathrm{e}}=.30$. As should be expected, the type of cue word presented on cued-recall tests did not affect recall on the uncued test. On the cued tests, however, presenting cues adjacent to the first presentation of the first lag exemplar and cues adjacent to the first presentation of the second lag exemplar resulted in better recall than any other combination of cues. Neither of these interactions presents serious complications, since the factors did not interact with any other variable.

The only other significant effect was the Lag $\times$ Cuing interaction, $F(2,72)=6.64$, $M S_{\mathrm{e}}=.22$. The single degree-of-freedom contrast comparing recall at Lags 2 and 17 to Lag 5, across cuing conditions, was significant, $F(1,72)=4.03$. Cued recall produced an inverted U-shaped spacing function, while uncued recall produced a monotonically increasing spacing function.

A difference between the results of Experiment 1 and those reported by Glenberg (1976) concerns the main effect of cuing. In the previous experiments, those conditions which led to nonmonotonic lag effects also produced superior overall performance compared to the conditions which led to monotonic effects. The lack of a main effect for cuing condition in this experiment was not totally unexpected. Slamecka (1968, 1969) reported a series of experiments using input items as retrieval cues in free recall. $\mathrm{He}$ found no facilitating effects of the retrieval cues as compared to uncued recall. One interpretation of this finding (Roediger, 1973 ) is that the retrieval cues only increase recall to the extent that they allow access to more subjective units than could be recalled without the cues. Roediger also suggests that "when more retrieval cues than are needed to produce access to higher-order units are provided ... recall of items from the higher-order unit will be impaired" (Roediger, 1973, p. 645). This interpretation is very similar to Rundus's (1973) interpretation of Slamecka's results. While the results of Experiment 1 are in general agreement with these theories, the theoretical positions themselves are orthogonal to the interpretation of the critical Lag $\times$ Cuing interaction.

In terms of the present assumptions, the lack of a significant main effect for cuing in Experiment 1 is easily explained. The predictions of the interaction between lag and cuing condition were based on the relation of the retrieval cues to the encodings developed at input. In Glenberg's (1976) study, the relationship of the cues to the input encodings was confounded with the effectiveness of the cue. These two factors were separated in Experiment 1. While the subjects used the cues to direct their recall, thereby insuring that the type of relationship needed to produce the nonmonotonic effect was present, the cue words were no more effective than the cues the subjects could generate on their own. The difference between the two cuing conditions is that the cues generated by the subject had no consistent bias toward the information stored at either the first or second presentation of the repeated words, resulting in a monotonic spacing effect.

\section{Experiment 2}

The interpretation of the results of Experiment 1 depended on the assumption that the subjects were forming subjective organizations or groups of interassociated items. These organizations produced the monotonic lag effect in free recall and allowed the cue words to direct retrieval in cued recall. Two implications of this explanation are that eliminating the organizations attenuates the lag effect and eliminates the interaction between cuing condition and lag. Experiment 2 was designed to test these predictions.

Half of the subjects in Experiment 2 were instructed to study the lists by using an organizational strategy. The strategy em- 
phasized associating groups of items. The other subjects were instructed to study by overtly rehearsing individual words on the lists, saying only the word being presented. This strategy should minimize the formation of interitem associations. Orthogonal to the strategy manipulation, half the subjects were given recall cues, while the other half were to free recall the words. The traditional, monotonic lag effect should be most pronounced for the subjects using the organizational strategy with free recall. Those subjects using the organizational strategy but given cued recall should generate the inverted $U$-shaped lag effect. For both groups of subjects using the repetition strategy, the lag effect should be attenuated and cuing condition should not interact with lag.

\section{Method}

Subjects. The subjects were 96 students at the University of Wisconsin-Madison. Of these students, 58 were obtained from introductory psychology classes as part of a course requirement. The remaining students were paid $\$ 2.50$ for participating in the experiment. The proportion of paid subjects was approximately the same in all conditions.

Materials and design. Each subject saw and recalled six lists of common four- and five-letter single-syllable nouns. The six lists were composed of two sets of three lists, reflecting the two sequences used in Experiment 1. The sequences were exactly like those used in Experiment 1, except that the primacy section of the lists was extended to six once-occurring words and the recency section was extended to eight once-occurring words.

The subjects were randomly assigned to one of the four groups created by the combination of two types of instructions (organization and single-word repetition) with the two cuing conditions (cued and free recall). Within each of these four groups, three subgroups were formed by counterbalancing the assignment of cue-words-repeated-word triples to specific lag conditions. Over subjects, each triple was used equally often in each lag condition. Within each of these subgroups, the subjects given cued recall received one of the four possible sets of cue words as described in the Method section of Experiment 1. For each subject in the cued-recall conditions, the order of the cues presented at recall was randomly arranged except for the first and second cue words, the last and first words of the list, respectively. The combination of the four groups, by counterbalancing and by cue words presented, calls for 48 subjects. A second group of 48 subjects was also run with the following changes: a different experimenter, a different sample of cuewords-repeated-word triples, and a different order of presentation of the six lists.
Procedure. Subjects were run individually. Upon entering the laboratory, the subjects were read one of four sets of instructions reflecting the four main conditions created by crossing learning instructions and cuing conditions. The instructions for the organization conditions gave examples of how to make associations between individual words, how to create stories, and how to form categories from the words on the list. The subjects in the repetition conditions were instructed to repeat each word out loud, four or five times, while it was being shown and to repeat only the word that was being shown. Those subjects who were to receive cued recall were told to try to use the cue words to help them recall other words from the list.

Each subject received a recall booklet containing seven pages and seven cover sheets. The first page was used for a practice sheet. The practice list was used to give the subjects practice using their respective learning strategies and recall procedures. The stimuli were projected via a Kodak Carousel projector controlled by an external timing apparatus. The stimuli were projected for $3 \mathrm{sec}$ each (including the amount of time needed to change slides). The subjects were allowed $2.5 \mathrm{~min}$ for recall following each list. At the end of each session, the subjects were asked if they followed the learning and testing instructions.

\section{Results and Discussion}

A total of 14 subjects admitted they did not follow the instructions. These subjects were eliminated from the data analysis. In order to preserve the counterbalancing, an additional 10 subjects were eliminated. These 10 subjects were chosen randomly under the constraints that they be drawn from the proper counterbalancing subgroups. The final design consisted of 72 subjects, 36 in each replication. Within each replication, there were 9 subjects in each learning strategycuing condition; within each of these 9 , groups of 3 subjects had different assignments of word triples to lag conditions.

Mean recall performance of the repeated words is displayed in Figure 1. The data in Figure 1 were analyzed in a $2 \times 3 \times 4 \times 2$ $\times 3$ (Replication $\times$ Counterbalancing of Word Triples $\times$ Learning Strategy and $\mathrm{Cu}$ ing $\times$ List $\times$ Lag) analysis of variance, with the last two factors being within subjects. The analysis revealed significant main effects for learning strategy, $F(1,48)=7.52, M S_{\mathrm{e}}$ $=4.89$; cuing condition, $F(1,48)=4.37$, $M S_{\mathrm{e}}=4.89 ;$ and lag, $F(2,96)=7.52, M S_{\mathrm{e}}$ $=77$. The critical term is the three-way 
interaction involving learning strategy, cuing, and lag. The six degrees of freedom in this term were broken down into orthogonal contrasts. The contrast comparing recall at Lag 5 to recall at Lags 2 and 17 in the organized study conditions to the two repetition study conditions was not significant. The contrast comparing recall at Lag 2 to 17 in the two organized conditions compared to the repetition conditions was significant, $F(1,96)=$ 4.62, $M S_{\theta}=.77$. These analyses indicate that recall of the Lag 17 item, compared to the Lag 2 item, was affected by cuing in the organized study conditions but not affected by cuing in the repetition study conditions. In effect, the shape of the lag function depended on the presence of retrieval cues only in the organized study conditions.

A number of other interactions were significant, although none affect the main conclusions. List type interacted with lag, $F(2$, 96) $=9.9, M S_{e}=1.32$. In the lists in which the second Lag 5 exemplar was presented toward the end of the list, recall of the $\operatorname{Lag} 5$ items was superior to the Lag 2 items. In the lists in which the second Lag 2 exemplar was presented toward the end of the list, the opposite result was obtained. The counterbalancing of cue-words-repeated-word triples to lag conditions interacted with lag, $F(4$, 96) $=2.94, M S_{\mathrm{e}}=.77$, as well as with replications and lag, $F(4,96)=4.18, M S_{\mathrm{e}}$ $=77$. In the first replication, the sets of word triples did not affect the shape of the lag function. In the second replication, one set of word triples accentuated the recall of the lag condition to which it was assigned. Finally, there was a significant interaction between replications, counterbalancing, list types, and lag, $F(4,96)=4.32, M S_{\mathrm{e}}=1.32$, which does not lend itself to verbal description.

The results of this experiment lead to a number of conclusions. First, recall is superior under instructions to organize than under instructions to repeat single items, The result supports the distinction between Type 1 (repetition) and Type 2 (associative) rehearsal made by Craik and Watkins (1973) and by Woodward, Bjork, and Jongeward (1973). Type 2 rehearsal is deeper, in the sense of Craik and Lockhart (1972),

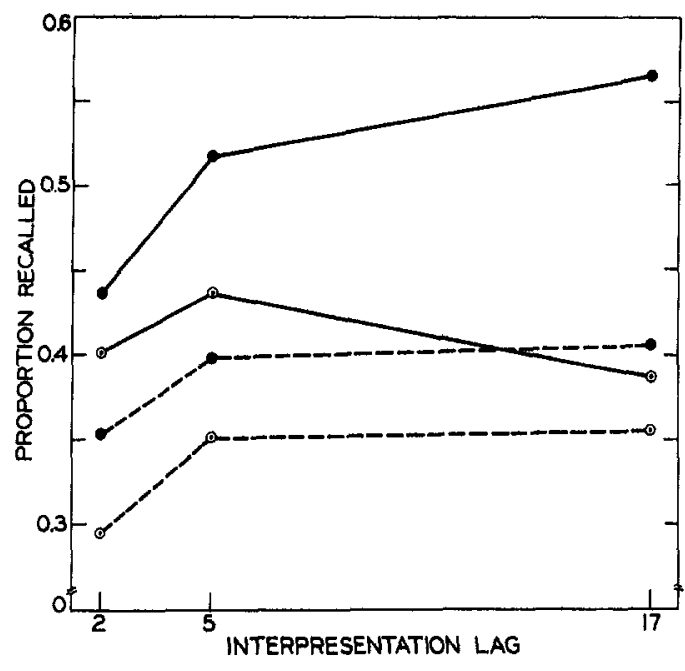

Figure 1. Mean recall of the repeated items in Experiment 2. (Solid lines = organizational strategy; dashed lines $=$ repetition strategy $;$ solid symbols $=$ uncued recall ; open symbols $=$ cued recall.)

and leads to better recall than Type 1 rehearsal.

Second, as suggested by Rundus (1973), cuing with items presented on the input list is detrimental to recall. The between-subjects design of Experiment 2 is probably responsible for the significant main effect of cuing condition which was not found in Experiment 1. Most of the subjects in Experiment 1 indicated that the cues provided at recall were more distracting than helpful, when compared to the opportunity to recall without the cues. Some of the subjects in Experiment 1 probably ignored the cues, which reduced the cuing main effect. In Experiment 2 , those subjects who received cued recall did not have the opportunity to compare cued and free recall. In addition, those subjects who did admit ignoring the cues were eliminated in Experiment 2, which should have increased the size of the cuing effect.

Third, as indicated in Figure 1, the most robust lag effect is found when subjects are free to organize the list of items (and not cued), supporting differential organization theories of the lag effect. In the repetition conditions, the lag effect is attenuated. These results are similar to those reported by D'Agostino and DeRemer (1973). In their 
Experiment 2, they demonstrated that decreasing the opportunity for differential organization (image same condition) attenuated the lag effect after Lag 5. They attributed the increase in the lag function up to Lag 5 to stimulus encoding variability, rather than to differential organization. This concept seems to provide an adequate explanation for the similar increase, in the repetition conditions, in the present Experiment 2.

Finally, the interaction between cuing condition and lag is found only when subjects organize the list items. This result provides good cause for considering the relationship between the information stored at input and the retrieval environment as a potent determiner of the shape of the lag function.

\section{Experiment 3}

Recently, Foos and Smith (1974) reported a nonmonotonic lag effect in free recall. In two of their conditions (twice-presented items and thrice-presented items with the same spacing between the first and second and between the second and third presentations), the lag function first increased and then decreased. In their twice-presented conditions, the average serial positions of the last presentations of the lag exemplars were equated across lag conditions. They maintained that the difference between their results and previously reported results of monotonic lag effects was due, in part, to careful control of the serial positions in which the lag exemplars were presented. Experiment 3 was designed to provide exact control of the serial positions of the last presentations by comparing the recall of items repeated at different lags, but presented in different lists, so that the last presentations were in the same serial positions. Across different lists, words repeated at lags of $0,2,8$, and 20 each had their last presentations in Serial Positions $27,31,33$, and 37 of a 42-item list.

The between-list manipulation also provides for a different test of the theory outlined in the introduction. The theory states that biasing retrieval toward one (but not both) of the sets of the groups associated with a word presented at a long lag produces the nonmonotonic lag effect. In Experiments 1 and 2 , this retrieval direction was provided by presenting words from the input list as cues. In Experiment 3, the bias is provided by the subjects' own retrieval strategies.

Tulving (1968) has suggested that one explanation for the recency effect in free recall is that subjects have available, at the end of the list, a multiplicity of retrieval cues that enhance the recall of the last few items. If this is a reasonable explanation for the recency effect, the lag function generated by those items repeated toward the end of the list (in Serial Position 37) should be nonmonotonic. The cues that generate the recency effect should provide access to groups of items presented near the end of the list. Since a word repeated (in Serial Position 37) after a moderate lag should be associated with more of the groups near the end of the list than a word repeated after a short lag, the word repeated after a moderate lag should be recalled more often. A word repeated in Serial Position 37 after a long lag (only one presentation is near the end of the list) should be associated with fewer end-of-the-list groups than the word repeated after a moderate lag. Again, the word repeated after a moderate lag should be recalled more often, generating the nonmonotonic lag effect. The lag function should become increasingly monotonic the further from the end of the list the items are repeated (Serial Positions 33, 31, and 27), since these items will not be strongly affected by the end-of-the-list cues.

\section{Method}

Subjects. There were 32 subjects in Experiment 3. Nine of the subjects were paid $\$ 2.50$ for their participation. The remaining subjects were recruited from introductory psychology classes at the University of Wisconsin-Madison. The subjects were also given a bonus based on the number of words recalled.

Materials and design. Four list types were used. Each list type was composed of 42 positions. The first 5 and the last 5 positions were filled with once-occurring items. Within the body of the list were 16 once-occurring filler items and 4 noncritical repeated items, 1 at each presentation lag of $0,1,2$, and 5 . These noncritical repeated items were confined to Serial Positions 6 to 25. Finally, each list had 4 repeated items, 1 at each lag of 0 , 2,8 , and 20 . The last presentations of these items 
occurred in Serial Positions 27, 31, 33, and 37. Over the four list types, each lag condition had its last presentation in each of the 4 serial positions. Eight lists, two of each type, were constructed from a pool of 272 single-syllable, common fourand five-letter nouns. Each subject saw each of the eight lists. The order of the eight lists was changed between groups of subjects so that each list type appeared equally often in each position.

The main experimental conditions conform to a $4 \times 4$ factorial design, with interpresentation lag as the first factor and serial position of the second presentation as the second factor. Each subject contributed to all 16 conditions. Across the 32 subjects a given word was assigned to 8 of these 16 conditions. This counterbalancing scheme allowed for the unambiguous comparison of the lag functions generated when the second presentations were in Serial Positions 27 and 37; and a separate comparison of the lag functions generated in Serial Positions 31 and 33.

Procedure. The subjects were run individually. After being read general instructions, the subjects were presented a 20 -item practice list which included 3 repeated items, 1 at each lag of 0,2 , and 5 . Following the practice list and a question period, the subjects were tested on the eight lists. Each word was projected with a Kodak Carousel projector for $3 \mathrm{sec}$ (including the time needed to change slides). After each list, the subjects were allowed 2 min for the recall of the words.

\section{Results and Discussion}

The percentages recalled of the noncritical repeated items and the once-presented items are as follows: noncritical repeated items, 39.6 ; primacy items, 44.9 ; filler items, 28.2 ; and recency items, 52.4 . The proportions recalled of the critical repeated items are presented in Figure 2. The recall of the critical repeated items was analyzed in two analyses of variance, one for Serial Positions 27 and 37 and one for Serial Positions 3.1 and 33. Each analysis was an $8 \times 4 \times 2$ (Counterbalancing of Words $\times \operatorname{Lag} \times$ Serial Position of the Last Presentation) analysis, with the last two factors being within subjects. For the analysis of the items presented in Serial Positions 31 and 33, the only significant term was for the lag variable, $F(3,72)=4.26$, $M S_{e}=.40$. In light of this result, these two serial positions were collapsed for presentation in Figure 2.

The analysis of Serial Positions 27 and 37 revealed a number of effects. The lag variable was significant, $F(3,72)=8.21, M S_{\mathrm{e}}=.46$, as was the effect of serial position, $F(1,24)$

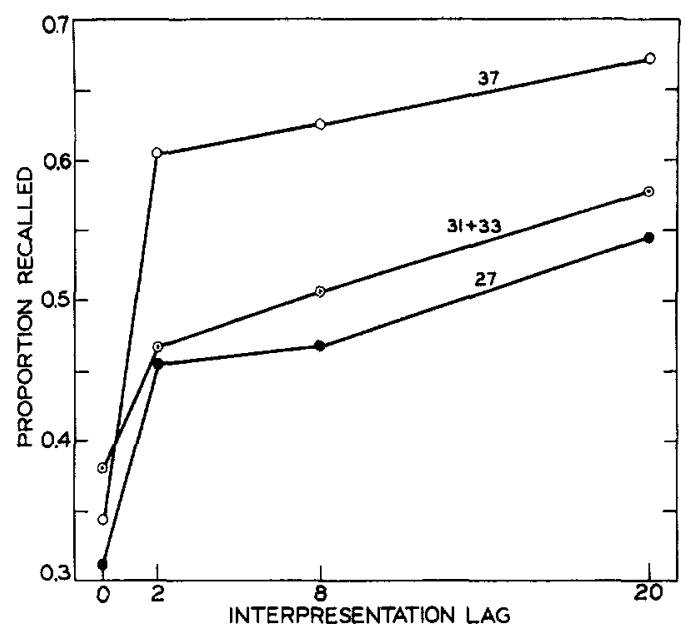

Figure 2. Mean recall of the repeated items in Experiment 3 . (The curve parameters indicate the serial position of the last presentations of the items.)

$=11.45, M S_{\mathrm{e}}=.31$. The Counterbalancing $\times$ Lag interaction was also significant, $F(21$, $72)=1.74, M S_{\mathrm{e}}=.464$. This interaction would present interpretive difficulties only if the Lag $\times$ Serial Position interaction or the Lag $\times$ Counterbalancing $\times$ Serial Position interaction were significant. For the former, $F(3,72)=.713$, and for the latter, $F(21$, $72)=1.36, M S_{\mathrm{e}}=.314, p>.10$ for both.

From these results it is clear that the monotonic lag effect is not an artifact of serial position. This finding stands in contrast to Foos and Smith's (1974) report of a nonmonotonic lag effect. It is unlikely that Foos and Smith's results were due to their control over the serial positions of the last presentation of the repeated items. In addition to the evidence from Experiment 3, Melton and Glenberg (Note 1) have found that in a long list of words with items repeated at lags of up to 80 intervening items (a) the lag effect is monotonic even when the serial positions of the last presentations are well controlled up to Lag 20 and (b) at all comparable serial positions, items repeated after long lags are recalled more often than items repeated after short lags. Currently, there does not appear to be a consistent explanation of both Foos and Smith's findings and these other results. 
Contrary to the prediction, the lag function was monotonic for items repeated in Serial Position 37. The prediction may have failed either because the subjects did not use the cues available at the end of the list to differentially access groups formed at the end of the list or because items presented in Serial Position 37 were not included in these groups. The prediction is tested again in Experiment 4, in which the lists contain a shorter recency buffer and the subjects were directed to recall items presented at the end of the list first.

\section{Experiment 4}

\section{Method}

The 64 subjects were drawn from the same source as for Experiment 3. The materials and design were exactly the same as in Experiment 3 except for the following: (a) The last three words were deleted from each list; (b) 64 subjects were used to allow for complete counterbalancing of words to conditions; (c) the subjects were instructed to recall the last few words on the list first; and (d) a new experimenter was used.

\section{Results and Discussion}

The proportions of noncritical items recalled are as follows: noncritical repeated items, .46; primacy items, .42; filler items,

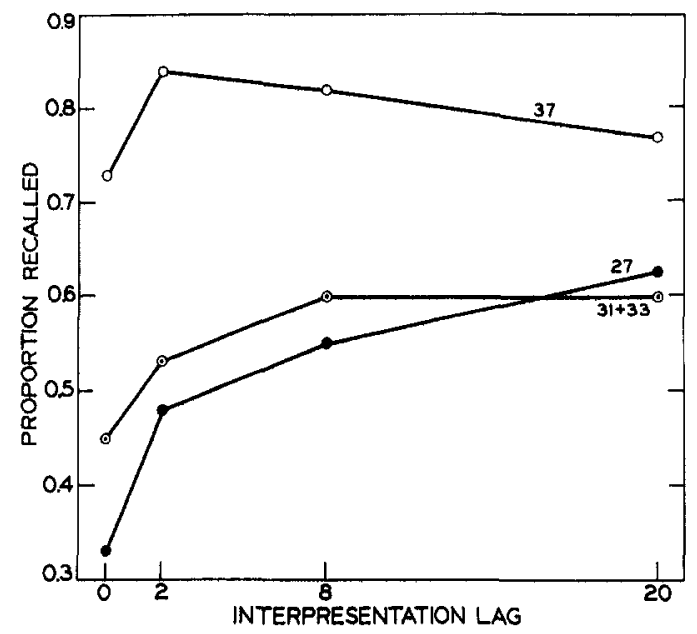

Figure 3. Mean recall of the repeated items in Experiment 4. (The curve parameters indicate the serial position of the last presentations of the items.)
.33 ; and recency items, .83 . While scoring the critical repeated items, it was discovered that one item was presented in two different lists. The data were analyzed both including and excluding the second presentation of this item. The pattern of significant effects and the absolute levels of recall were essentially identical in both analyses. The results based on the exclusion of the repeated items are presented in the remainder of this article.

The proportion of critical repeated items recalled is presented in Figure 3 . These results were analyzed in a $16 \times 4 \times 3$ (Counterbalancing $\times$ Lag $\times$ Positions of the Last Presentation) analysis of variance. The recall of the items in Positions 31 and 33 appeared to be the most variable (in terms of the shape of the lag function) and recall from those two levels of the position factor were collapsed before the analysis.

The main effects for lag, $F(3,144)=$ 12.81, $M S_{\mathrm{e}}=.34$, and position of the last presentation, $F(2,96)=64.51, M S_{\mathrm{e}}=.36$, were significant. The most important term is the Lag $\times$ Serial Position interaction. This effect was also significant, $F(6,288)=2.96$, $M S_{\mathrm{e}}=.33$. As indicated in Figure 3, the lag effect is monotonic at Serial Position 27 and is nonmonotonic at Serial Position 37. The Counterbalancing $\times$ Serial Position $\times$ Lag interaction was also significant, $F(90,288)$ $=1.40, M S_{\mathrm{e}}=.33$. Fortunately, this interaction need not affect the main conclusions of the experiment. In 11 of the 16 counterbalancings, the lag effect generated by the items in Serial Position 27 was monotonically increasing. In 13 of the 16 counterbalancings, the lag effect generated by the items in Position 37 was nonmonotonic; recall of the Lag 20 items was less than recall of the Lag 8 or Lag 2 items. The major contribution to the triple interaction was provided by the items in Positions 31 and 33. For these positions, in half of the counterbalancings the lag effect was monotonic, while the effect was nonmonotonic in the other half.

We can view these results as a replication of Experiment 1. In that experiment, retrieval direction was provided by giving subjects input words as retrieval cues. The cues were designed to bias retrieval toward the 
items associated with either the first or the second presentation of the Lag 17 word, but not both. The retrieval bias resulted in the nonmonotonic lag effect. In Experiment 4, the retrieval direction was provided by instructing subjects to recall the last few words of the list first. This direction should have biased retrieval toward the groups associated with the second presentation of the Lag 20 word presented in Serial Position 37. Again, the nonmonotonic lag effect was produced.

It would seem unlikely that all of a subject's retrieval efforts could be controlled by the simple instruction to recall the last few words of the list first. We might hypothesize that a subject's retrieval was directed by two sets of cues with the first set directing the subject toward the end of the list. The second set of cues would be those that the subject could generate that would lead to recall of other items on the list. The theory predicts the nonmonotonic lag effect only for the words recalled with the retrieval direction provided by the first set of cues. Only these cues should provide greater access to the words repeated at moderate lags compared to the words repeated at Lag 20. Retrieval with the second set of cues should result in approximately equal access to all groups. Since the number of groups in which a word is represented increases monotonically with lag, the second set of cues should generate the monotonic lag effect. Finally, the theory predicts that the words recalled using the first set of cues should result in a nonmonotonic lag effect regardless of the serial position in which the words were originally presented. The theory derives its predictions from the relationship of the retrieval cues to the internal representation or organization of the list of words. Typically, words presented in one part of a list are organized with other adjacent, or nearly adjacent, words. This was, of course, the reasoning that led to the predictions for Experiments 1 and 2. Nonetheless, it is unlikely that the correlation is perfect. Some items presented in early serial positions may be represented in portions of the internal structure that are easily accessed by the end- of-the-list retrieval cues. For these items, the lag effect should be nonmonotonic. The words recalled with the second set of cues should generate a monotonic lag effect, regardless of their actual input position.

To test these predictions, the subjects' recall protocols were divided into two sections corresponding to the words recalled with the first set of cues and the second set of cues, respectively. It was assumed that the first words recalled would be based on the first set of cues and that the remaining words would be recalled via the second set of cues. To find an appropriate dividing line, three subjects were chosen and their 24 recall protocols (eight lists per subject) were inspected. It appeared that the first third of their recalls came predominately from the end of the presentation list, while the remaining two thirds of the words recalled were distributed throughout the whole presentation list. Based on these observations, each recall protocol was divided into two sections, the first third of the words recalled and the remaining words. The words recalled in the first third of the protocol correspond to words retrieved with the end-ofthe-list cues. The remaining recalls correspond to the words retrieved with the second set of cues.

The data from the first and second sets of cues are presented in Figure 4. The ordinate indicates the absolute number of recalls, not proportion recalled. The absolute number of recalls seemed preferable, since there are a number of choices as to the proper divisor. Whether the proportions are based on the total opportunities for recall or the opportunities corrected for the number of items already recalled, the curves retain the approximate shapes depicted in Figure 4. Note that the lag function is nonmonotonic for the words recalled using the first set of cues, irrespective of the input serial position.

The data in Figure 4 were analyzed in two separate analyses of variance, one for the words recalled with the first set of cues and one for the words recalled with the second set of cues. For the first set of cues, the main effects of lag, $F(3,144)=4.59, M S_{\mathrm{e}}=.30$, 


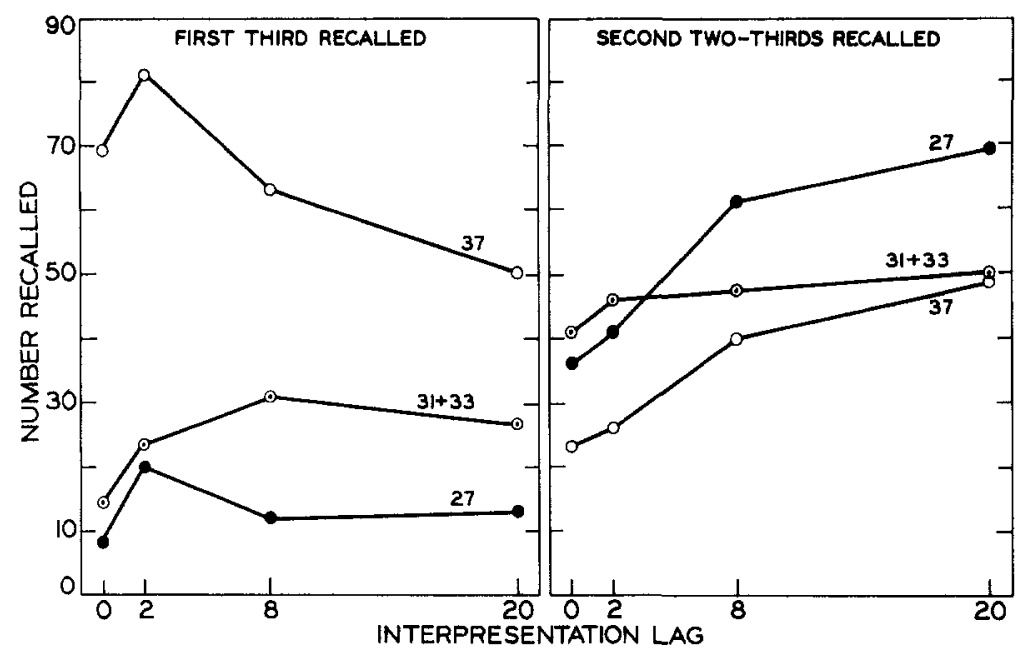

Figure 4. Absolute number recalled of the repeated items in Experiment 4. (The curve parameters indicate the serial position of the last presentations of the items. Left panel: Words recalled in the first third of the recall protocols. Right panel: Words recalled in the second two thirds of the recall protocols. For both panels, the total words recalled from Serial Positions 31 and 33 was divided in half before plotting.)

and serial position, $F(2,96)=164.13, M S_{\mathrm{e}}$ $=.30$, were significant. The lag main effect was due predominately to the contrast comparing recall at Lags 2 and 8 to recall at Lags 0 and 20, $F(1,144)=9.93, M S_{\mathrm{e}}=$ .30. This contrast did not interact with serial position, indicating that across the serial positions, the lag function was nonmonotonic. There was a significant interaction between lag and serial position, however, which was due predominately to the variation in the difference between the recalls of Lags 0 and 20 across the three serial positions, $F(2$, 288) $=7.45, M S_{\mathrm{e}}=.25$. Finally, there was a significant interaction between counterbalancing and serial position, $F(30,96)=$ 2.02, $M S_{\mathrm{e}}=.30$. Although recall increased monotonically with serial position in all of the counterbalancings, the degree of increase varied unsystematically.

The analysis of the words recalled in the second two thirds of the recall protocols revealed significant main effects for serial position, $F(2,96)=11.30, M S_{\mathrm{e}}=.43$, and lag, $F(3,144)=12.07, M S_{\mathrm{e}}=.43$. As indicated in the right-hand panel of Figure 4, these spacing functions are monotonically increasing regardless of the input serial positions. In addition, there is no statistical sup- port for an interaction between lag and serial position.

An anonymous reviewer has suggested that the nonmonotonicity found in this experiment is due to the recency effect in free recall. Two representations of the closely spaced items may have occurred within the range of the recency effect, but only a single representation of the widely spaced items occurred in this range. Hence the closely spaced repetitions may be selectively influenced by whatever causes the recency effect resulting in the nonmonotonic lag effect. This explanation does not, however, account for the increase in recall between Lag 0 and Lag 2. Nevertheless, this partial explanation is similar to the interpretation of the nonmonotonicity offered in this article. The twoprocess theory maintains that selectively enhancing the retrievability of one portion (group) of the internal structure that contains a representation of a repeated item produces the nonmonotonicity. A word repeated after a moderate lag interval is associated with more of the selectively enhanced groups than a word repeated after a longer interval or a shorter interval (because of differential organization), resulting in greatest recall of the moderate lag item 
and the nonmonotonic lag effect. In Experiments 1 and 2 , this selective enhancement was provided by directing retrieval with cues taken from the input list. In Experiment 4 , the selective enhancement was provided by taking advantage of the greater accessibility of the items presented at the end of the list, that is, the recency effect. The only difference between the experiments, as far as the theory is concerned, is that the cue words in Experiments 1 and 2 were not particularly strong cues. They served to modify the subjects' retrieval strategies but did not improve recall. In Experiment 4, the instruction to recall the last-presented items first elevated the recall of the repeated items (compared to Experiment 3), as well as modifying, or at least taking advantage of, the subjects' retrieval strategies.

\section{General Discussion}

Experiments 1-4 disconfirm some theories of spacing effects. The General Forgetting Theory (Bjork, Note 2) can be used to predict the monotonic lag effect and, with certain parameters, it can generate a nonmonotonic lag effect (Sperber, Greenfield, \& House, 1973). The theory, however, predicts the nonmonotonic lag effect as a result of changes in the storage characteristics of the repeated word. The theory cannot explain changes in the shape of the lag effect. produced by manipulating the retrieval environment. The same criticism applies to Rundus's (1971) rehearsal explanation for the lag effect, various explanations based on attention, and Atkinson and Shiffrin's (1968) explanation for the nonmonotonic effect. Hintzman's (1974) habituation theory specifically excludes the nonmonotonic lag effects from consideration, relegating the nonmonotonicity to processes other than those in volved in the monotonic lag effect. The research presented in this article and in Glenberg (1976) clearly contraindicates this piecemeal strategy.

In regard to the two-process theory of spacing effects presented in the introduction, three major findings from this set of experiments are important. First, the lag effect is most robust when subjects use an organiza- tional strategy (Experiment 2), suggesting that lag effects are a function of differential organization. Second, using cue words to direct retrieval to one area of the internal representation results in a nonmonotonic lag effect (Experiment 1), but only when subjects have imposed some structure on the list (Experiment 2). This finding suggests that the shape of the lag effect is controlled by the nature of the retrieval cues used by the subjects. Third, the nonmonotonicity can be produced in the absence of cue words by directing subjects' retrieval with instructions (Experiment 4). The important manipulation is not the use of cue words themselves, but in differentially enhancing the accessibility of certain portions of the internal representation of the list.

The present theory can be compared to Glenberg's (1976), in which a similar theory was proposed to account for monotonic and nonmonotonic lag effects in the continuous paired-associate and recognition memory paradigms. The major difference between the two theories is in the unit of analysis. For free recall the theory assumes that the monotonic lag effect is the result of differentially increasing the number of retrieval routes (groups) to the words repeated after long lags. In the paired-associate paradigm the monotonic effect is also produced by increasing the number of retrieval routes, but in this case the retrieval routes are assumed to be different encodings of the stimulus term of the paired associate. The conditions at the test can then influence the relative availability of the different stimulus encodings. A monotonic lag effect is produced when encodings of the stimulus are equally accessible; a nonmonotonic effect of lag results when some encodings are made differentially accessible. In both paradigms the same general mechanisms can be used to explain spacing effects and to delimit the conditions that control the effectiveness of any repetition. First, a repetition is potentially effective to the degree that different retrieval routes are established during input. Second, the retrieval cues used at the time of testing determine the extent to which that potential is realized. 


\section{Reference Notes}

1. Melton, A. W., \& Glenberg, A. M. An analysis of the free recall spacing effect using the overt rehearsal technique. Unpublished manuscript, 1972. (Available from Arthur Glenberg, Department of Psychology, University of Wisconsin, Madison, Wisconsin 53706.)

2. Bjork, R. A. Learning and short-term retention of paired-associates in relation to specific sequences of interpresentation intervals (Tech. Rep. No. 106). Palo Alto, Calif.: Stanford University, Institute for Mathematical Studies in the Social Sciences, 1966.

\section{References}

Anderson, J. R. FRAN : A simulation model of free recall. In $\mathrm{G}$. $\mathrm{H}$. Bower (Ed.), The psychology of learning and motivation (Vol. 5). New York: Academic Press, 1972.

Atkinson, R. C., \& Shiffrin, R. M. Human memory : A proposed system and its control processes. In K. W. Spence \& J. T. Spence (Eds.), The psychology of learning and motivation: Advances in research and theory (Vol. 2). New York: Academic Press, 1968.

Buschke, $H$. Learning is organized by chunking. Journal of Verbal Learning and Verbal Behavior, $1976,15,313-324$.

Craik, F. I. M., \& Lockhart, R. S. Levels of processing: A framework for memory research. Journal of Verbal Learning and Verbal Behavior, $1972,11,671-684$

Craik, F. I. M., \& Watkins, M. J. The role of rehearsal in short-term memory. Journal of Verbal Learning and Verbal Behavior, 1973, 12, 599-607.

D'Agostino, P. R., \& DeRemer, P. Repetition effects as a function of rehearsal and encoding variability. Journal of Verbal Learning and Verbal Behavior, 1973, 12, 108-113.

Foos, P. W., \& Smith, K. H. Effects of spacing and spacing patterns in free recall. Journal of Experimental Psychology, 1974, 103, 112-116.

Glenberg, A. M. Monotonic and nonmonotonic lag effects in paired-associate and recognition memory paradigms. Journal of Verbal Learning and Verbal Behavior, 1976, 15, 1-15.
Hintzman, D. L. Theoretical implications of the spacing effect. In R. L. Solso (Ed.), Theories in cognitive psychology: The Loyola Symposium. Potomac, Md.: Erlbaum, 1974.

Madigan, S. A. Intraserial repetition and coding processes in free recall. Journal of Verbal Learning and Verbal Behavior, 1969, 8, 828-835.

Melton, A. W. The situation with respect to the spacing of repetitions and memory. Journal of Verbal Learning and Verbal Behavior, 1970, 9, 596-606.

Roediger, H. A., III. Inhibition in recall from cueing with recall targets. Journal of Verbal Learning and Verbal Behavior, 1973, 12, 644-657.

Rundus, D. Analysis of rehearsal processes in free recall. Journal of Experimental Psychology, 1971, 89, 63-67.

Rundus, D. Negative effects of using list items as recall cues. Journal of Verbal Learning and Verbal Behavior, 1973, 12, 43-50.

Slamecka, N. J. An examination of trace storage in free recall. Journal of Experimental Psychology, $1968,76,504-513$.

Slamecka, N. J. Testing for associative storage in multitrial free recall. Journal of Experimental Psychology, 1969, 81, 557-560.

Sperber, R. D., Greenfield, D. B., \& House, B. J. A nonmonotonic effect of distribution of trials in retardate learning and memory. Journal of $E_{x}$ perimental Psychology, 1973, 99, 186-198.

Tulving, E. Subjective organization in free recall of unrelated words. Psychological Review, 1962, $69,344-354$.

Tulving, E. Theoretical processes in free recall. In T. R. Dixon \& D. L. Horton (Eds.), Verbal behavior and general behavior theory. Englewood Cliffs, N.J.: Prentice-Hall, 1968.

Underwood, B. J. Some correlates of item repetition in free recall learning. Journal of Verbal Learning and Verbal Behavior, 1969, 8, 83-94.

Wallace, W. P. Clustering in free recall based upon input contiguity. Psychonomic Science, 1969, 14, 290-292.

Woodward, A. E., Jr., Bjork, R. A., \& Jongeward, R. H., Jr. Recall and recognition as a function of primary rehearsal. Journal of Verbal Learning and Verbal Behavior, 1973, 12, 608-617.

Received August 19, 1976

Revision received October 14, 1976 